\title{
Was bringt die Blutzuckerselbstmessung bei Kindern und Jugendlichen mit Diabetes?
}

\section{Die Blutzuckerselbstmessung (BZSM) ist ein wichtiger Baustein in der} effektiven Behandlung von Kindern mit Diabetes. Es gibt aber nur wenige Studien, die untersuchen, wie Messfrequenz und Stoffwechselkontrolle sowie Komplikationen zusammenhängen. Auch der Einfluss der Therapieform ist in diesem Alter nicht bekannt.

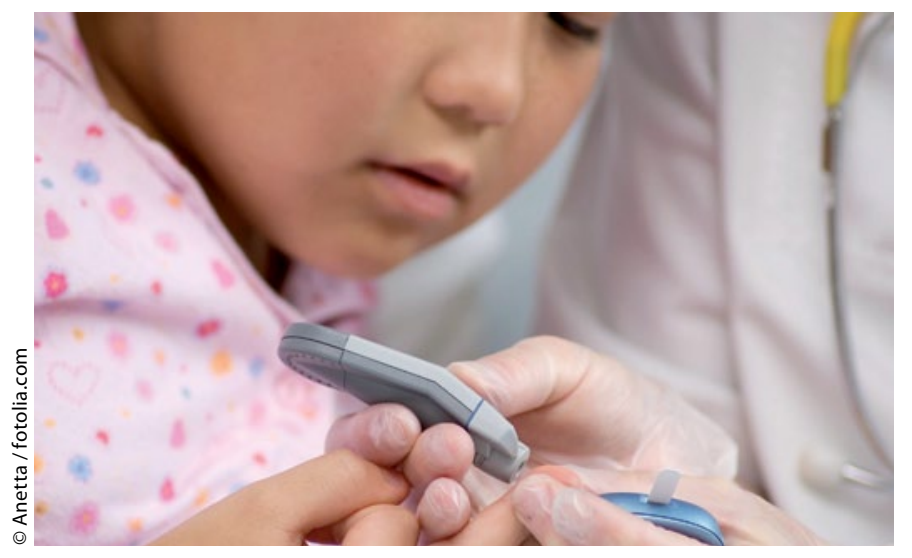

Fünf Blutzuckerbestimmungen pro Tag sind optimal.

n einer Studie wurden die Daten der DPV-Datenbank aus den Jahren 19952006 analysiert. In dieser Zeit wurden 26.723 Kinder und Jugendliche in 233 deutschen und österreichischen Zentren betreut. Für die Studie wurden für jeden Patienten Durchschnittswerte aus dem aktuellsten Therapiejahr verwendet. Außer der Zahl der BZSM wurden Geschlecht, Alter, Diabetesdauer, Ketoazidose mit stationärer Aufnahme, Hypoglykämien und $\mathrm{HbA}_{1 \mathrm{c}}$ (mathematisch standardisiert) erfasst. Es erfolgte eine Subanalyse von drei Altersgruppen (0-5 Jahre: 7,4\%, 6-12 Jahre: 28,3\%, 13-18 Jahre: $64,2 \%)$. Das mittlere Alter lag zuletzt bei 12,7 Jahren, die mittlere Diabetesdauer bei 4,8 Jahren.

Der mittlere $\mathrm{HbA}_{1 \mathrm{c}}$ lag insgesamt bei $8,16 \%$ und war vom Alter $(0-5$ Jahre $=$ 7,59\%, 6-12 Jahre = 7,61 \%, 13-18 Jahre $=8,46 \%$ ) und der Therapieform abhängig. Im Mittel wurde von den Patienten oder deren Eltern der Blutzucker 4,7-mal pro Tag gemessen. Von 1995 bis 2006 stieg diese Zahl kontinuierlich von 3,2 auf 5,1 BZSM/d. Dabei wurde am meisten im Kleinkindesalter gemessen (6) und am wenigsten von den Jugendlichen $(4,4)$. Pumpenpatienten kontrollierten ihre Werte signifikant häufiger (5,3/d vs. 4,7/d bei ICT).
Mehr Messungen waren signifikant mit einer besseren Einstellung assoziiert. Im Schnitt kann mit einer Messung mehr am Tag eine Verbesserung des $\mathrm{HbA}_{1 \mathrm{c}}$ um 0,2\% erreicht werden, mit Pumpe sogar um $0,27 \%$. Ab einer Messung von fünf Werten am Tag zeigte sich aber keine signifikante Verbesserung mehr.

Es gab auch eine signifikante Beziehung zwischen der Häufigkeit von Hypoglykämien und der BZ-Messfrequenz. Die Rate der Hypoglykämien stieg mit der Messfrequenz, für die Zahl von Ketoazidosen war die Beziehung invers. Es gibt also eine strenge Assoziation der BZSM-Häufigkeit (bei 0-5 Messungen/d) und der Stoffwechseleinstellung. Dies betrifft vor allem Jugendliche.

Ziegler R et al. Frequency of SMBG correlates with $\mathrm{HbA} 1 \mathrm{c}$ and acute complications in children and adolescents with type 1 diabetes. Pediatric Diabetes $2011 ; 12: 11-7$

Kommentar: Die Interpretation dieser Daten muss sehr vorsichtig erfolgen. Es zeigt sich aber eine klare Abhängigkeit der Zahl von BZSM mit der Stoffwechseleinstellung, gemessen am $\mathrm{HbA}_{1 \mathrm{c}}$ im Bereich von 0-5 Messungen pro Tag, bei der jede zusätzliche Messung den $\mathrm{HbA}_{1 c}$ verbessert. Jugend- liche scheinen von der Erhöhung ihrer Messfrequenz mehr zu profitieren, auch über fünf Messungen hinaus. Gerade in diesem Alter ist die Zahl der Messungen ein valider Parameter für die gute Selbstbehandlung des Diabetes, da sie ja eine Dosisanpassung nach der Messung erlaubt. Bei Kleinkindern machen die Eltern meist eine Therapie mit häufigen Messungen (4-7), sodass hier keine so gravierenden Unterschiede in Erscheinung treten. Gerade Pumpenpatienten profitieren aber auch von mehr Messungen, da eine zeitnahe Reaktion auf das Messergebnis ohne wesentlichen zusätzlichen Aufwand (Pen nehmen und spritzen) erfolgen kann.

Bei Patienten, die deutlich mehr messen, zeigt die Messfrequenz möglicherweise die momentan schwierige Stoffwechselsituation mit schwankenden Werten an. Auch die höhere Messfrequenz bei Hypoglykämien ist sicher nicht als kausaler Zusammenhang zu deuten. Bei bekannter Unterzuckerungsneigung wird man sicher öfter den Blutzucker kontrollieren, um ein erneutes Auftreten eines solchen Ereignisses zu vermeiden. Limitiert wird die Studienaussage dadurch, dass es sich um keine randomisierte Studie handelt. Dafür spiegeln die Daten aber das "real life" wider.

Dr. Thomas. M. Kapellen

Babys besser nachmittags impfen? Ärzte der University of California in San Francisco haben untersucht, wie sich verschiedene Parameter auf den Schlaf von Säuglingen nach der ersten Impfung auswirken. 70 Kinder im Alter von zwei Monaten erhielten die in den USA empfohlenen Vakzinen (Pneumokokken, DTP, Hib, Polio, HBV und z. T. Rotavirus). Die Ergebnisse: In den 24 Stunden nach der Impfung schliefen die Kinder im Durchschnitt 69 Minuten länger als in den 24 Stunden davor. Die Schlafdauer stieg vor allem bei Kindern, die nach 13.30 Uhr geimpft worden waren (+ 98 Minuten). Bei den früher am Tag Geimpften nahm sie weniger zu (+ 35 Minuten), und viele Kinder schliefen sogar kürzer als im Vergleichszeitraum. Dagegen hatte die prophylaktische Gabe von Paracetamol keinen Einfluss auf die Schlafdauer.

Dr. Beate Schumacher

Franck L et al. Pediatrics 2011; 128: 1100-8 